Full reference: Fullwood, C., Quinn, S., Kaye, L. K., \& Redding, C. (2017). My Virtual friend: A qualitative analysis of the attitudes and experiences of Smartphone users: Implications for Smartphone attachment. Computers in Human Behavior, 75, 347-355. doi: 10.1016/j.chb.2017.05.029

\title{
My Virtual friend: A qualitative analysis of the attitudes and experiences of Smartphone users: Implications for Smartphone attachment
}

\author{
Chris Fullwood ${ }^{1}$, Sally Quinn ${ }^{2}$, Linda K. Kaye ${ }^{3}$ \& Charlotte Redding ${ }^{1}$ \\ ${ }^{1}$ Institute of Psychology, University of Wolverhampton, UK \\ ${ }^{2}$ Department of Psychology, University of York, UK \\ ${ }^{3}$ Department of Psychology, Edge Hill University, UK
}

\begin{abstract}
As mobile phones have evolved into Smartphones, they have become more than simple communication tools; transforming into personal assistants, entertainment devices and information gateways. There is a need to understand how this rapid transformation and complexity of Smartphone uses have impacted on users' relationship with their phones. This study presents a thematic analysis of three focus group discussions around attitudes and experiences of owning and using Smartphones. Themes that emerged included a bifurcation in attitudes to Smartphones as simultaneously materialistic objects, and ones which users express anthropomorphic and sentimental views about. Participant accounts reflected the evolution of Smartphones from functional communication devices, to informational and recreational tools. Participants discussed using Smartphones to alleviate boredom and that device usage had become habituated for some users. However, context determined Smartphone use with some participants using them to feel secure while away from familiar settings. Participant accounts provide rich insights into different Smartphones uses and infer numerous implications for understanding why some users develop strong psychological attachments to them. Findings also imply that users may not be attached to the device itself, but rather the affordances on
\end{abstract}


offer. The implications of these findings, for example in the assessment of Smartphone addiction, are discussed.

Keywords: Smartphones, focus group, thematic analysis, attitudes, experiences, attachment. 


\section{Introduction}

Mobile phones have become an integral part of $21^{\text {st }}$ century living with ownership increasing over the last decade. For example, OFCOM (2016) report a rise in UK adult mobile phone ownership from $82 \%$ in 2005 to $90 \%$ in 2015. Mobile phones themselves have also developed, with the Smartphone now being widely available, associated with increased ownership of these more technical devices (OFCOM, n, d). Smartphones are powerful, Internet-enabled devices with user-friendly interfaces and advanced operating systems which afford their users many more features and facilities (e.g. downloadable 'apps', event calendars, digital cameras, GPS navigation and media players) than the traditional mobile phone (Wang et al., 2014). Indeed, this is pertinent when exploring the psychological impacts of these devices, in which research requires a much more refined account of how these specific affordances influence attitudes and behaviour. For example, earlier research suggested that mobile phone owners largely valued their phones for their instrumental value. For instance, Leung and Wei (2000) noted how users appreciated their mobile phones more for immediate access to contacts and communication on-the-go than for intrinsic reasons such as the phone helping them to feel relaxed. Walsh et al. (2008) found that users primarily felt the phone was important for being able to contact others and for being contactable. However, given the evolution of these devices into mobile personal computers, it is timely to explore more recent attitudes and experiences, to ascertain the impact that these developments may have had on the relationship that users have with their phones as well as any implications that this might have for helping to understand the reasons why some people develop a strong psychological attachment to these devices. This is particularly pertinent given the recent spate of academic literature focusing on the detrimental effects of Smartphone addiction. For example, Smartphone addiction has been associated with a number of negative effects on wellbeing and mental health (e.g. lower satisfaction with life, higher levels of stress) as well numerous dysfunctional behavioural patterns (e.g. poorer academic performance, lower physical activity) (e.g. see Choi et al,, 2012; Haug et al., 2015; Samaha \& Hawi, 2016). Thus, the present study aims to provide rich insights into current attitudes towards and experiences of using Smartphones by reporting on a qualitative thematic analysis of focus groups discussions probing young people's perceptions of these.

\subsection{A functional perspective of Smartphones}

Among the many functions which Smartphones serve, one key function which has been found across a number of studies is its communicational capacity (Aoki \& Downs, 2003; Chen \& 
Katz, 2009; Walsh et al 2008, 2009). Smartphones allow users to contact and be contacted by others while 'on-the-go' and this is often perceived to be a positive aspect of mobile phone ownership. For example, college students see their mobile phone as a particularly important tool for overcoming geographical distance and for keeping in touch with family (Chen \& Katz, 2009) and in a sample of Japanese undergraduate students Toda et al. (2006) found evidence to suggest that communicating with others via the mobile phone helps to alleviate stress. This may be partially due to its potential to allow users to gain greater control over some of their social interactions, due to the asynchronicity it offers in features such as text messaging (Madell \& Muncer, 2007). For example, users can reply to messages at their own convenience. The fact that instantaneous replies may not always be expected would also mean that users could spend more time thinking about how they want to construct their messages before sending them (Fullwood, 2015). This communicative aspect of mobile phones, through its ability to allow users to communicate "whenever and wherever", has resulted in some users feeling as though they are cut-off from their friends, missing out on something (Walsh et al 2008), or feeling lost (Tian et al., 2009) when they do not have their phone with them, thus suggesting a sense of dependence on the phone (Aoki \& Downs, 2003; Jarvenpaa \& Lang, 2005), which has been speculated to be a contributing factor to socalled mobile phone addiction (Kwon et al., 2013).

When it comes to the issue of mobile phone addiction, there has been significant debate in the academic literature regarding what the addictive element may actually consist of. Are users 'addicted' to the phone itself or to the varying affordances that the phone provides? This debate has become further intensified with the evolution of the mobile phone into the Smartphone and with the numerous features that these devices now offer (Lopez-Fernandez, 2015; Meschtscherjakov, 2009) and some researchers have even called for more qualitative work to be done to shed light on any additional factors which may contribute to explaining addictive tendencies (Van Deursen et al., 2015). In support of these ideas, research has found that mobile phone users have relatively low emotional attachments to the devices themselves, but high emotional attachment to the possible activities and services that it offers (Meschtscherjakov, 2009; Venta et al., 2008). Evidence also shows that using Smartphones for accessing social networking sites such as Facebook is a significant predictor of addiction to these devices (Salehan \& Negahban, 2013). This facility is not available on non-internet enabled phones and newer measures of Smartphone addiction are reflecting these changes (e.g. Kwon et al's Smartphone addiction scale, 2013). Psychological enquiry may therefore benefit 
from approaching these issues through the lens of functionality or a Uses and Gratifications (U\&G) framework (Katz et al., 1974), both of which may provide a more nuanced account of the types of functions that Smartphone users value as well as any potential implications that specific uses may have for developing an attachment to or dependence on these devices.

$\mathrm{U} \& \mathrm{G}$ theory is an audience-centred approach to explaining why individuals make specific media choices. The theory argues that people seek out specific media options in order to gratify very personal needs. Furthermore, satisfactorily gratifying these needs predicts continued engagement with these media options (Katz et al., 1974). In respect of a U\&G perspective, research has revealed that key motivations for Smartphone use relate to helping users to relax, escape problems, and alleviate negative mood and boredom (Pew Research Center, 2015; Smetaniuk, 2014). Thus, one might expect that users will not use their Smartphones uniformly, but rather will make use of very specific features to gratify personal needs. For example, the need to alleviate boredom may result in the use of more entertainment features (e.g. surfing the Internet, playing games). Furthermore, Joo and Sang (2013) argue for an integration of the U\&G and Technology Acceptance models in helping to explain Smartphone adoption and use. The Technology Acceptance Model (TAM; Davis, 1986) posits that adoption of new technology can be predicted by perceived ease of use (i.e. the level of effort required to adapt to the new technology) and perceived usefulness (i.e. the degree to which the technology may enhance and add value to the individual's life, for example in their job or studies). Joo and Sang (2013) argue that whereas TAM may be good at explaining extrinsic motivations for using Smartphones (e.g. helping the person to achieve better grades), U\&G addresses intrinsic factors (e.g. using the phone for entertainment or to feel good). Findings from their study suggest that Korean Smartphone users are more motivated by goal-oriented and instrumental factors than habitual and less-goal orientated motivations. In other words, purchasing decisions were primarily motivated by how useful they perceived the Smartphones to be, particularly with respect to the phone's features which might allow them to customise their Smartphone to meet their specific usage requirements and needs.

Research also suggests that there may be individual differences in how users make use of their Smartphones. For instance, two distinct types of user have been established in respect of their usage of different Apps and tools; "Instant Communicators" and "Communicators/Information Seekers" (Head \& Ziolkowski, 2012). "Instant Communicators" tend to place a larger emphasis on text messaging and taking photographs (ibid). For this group, factors such as ease of use, 
usefulness, enjoyment of using the phone and ability to express oneself, all influence positive attitudes towards the phone. Alternatively, "Communicators/ Information seekers", also perceive text messaging to be important, but place higher importance on email and webbrowsing facilities (ibid), which is arguably a more utilitarian approach to Smartphone usage. This approach is mirrored in other findings suggesting that users value the phone's ability to access information such as friends' phone numbers, online services such as banking, and as a time management tool (e.g. arranging to meet people; Aoki \& Downs, 2003; Pew Research Center, 2015). Thus, it is important to recognise some degree of specificity in how Smartphones function for different users and that they have developed into much more than communication devices.

\subsection{Me, My Phone and I}

As well as functionality as previously outlined, other features of Smartphones which are useful to consider from a psychological perspective, are customisation and personalisation. That is, research has found personalising phones, as well as being a device through which to store personal memories and thus reflecting an aspect of "the self", are key components towards the relationship a user has with their phone (Meschtscherjakov, 2009; Tian et al., 2009, Venta et al., 2008). However, it is not just an individual's personal identity that a phone can help express. There is also evidence to suggest that phones express aspects of our social identities, or the extent to which we define ourselves by our membership to specific groups (Tajfel \& Turner, 1979). For example, Walsh et al. (2009) found that in a sample of 16-25 year olds, the level and type of use of a mobile phone was an expression of social identity. Having a phone, the Phone Company used, and speed of response to messages were all indicators of group norms (i.e. the norms of the peer group). These findings are supported by other evidence of how Smartphones operate as a status symbol, particularly for younger users, which in turn manifests in peer status and popularity (Aoki \& Downs, 2003; Vanden Abeele et al., 2013). These data would support Srivastava's (2005) argument that some mobile phone owners see their devices as status symbols. The type of phone people own and the way they use it can therefore reflect aspects of people's identities, and thus hold an emotional connection via selfconceptualisation.

It is clear that understanding users' psychological experiences associated with their Smartphone are complex, and requires a perspective which accounts for the nuances of functionality, beyond their communicative capacity, which these devices afford. What is not 
yet clear in the literature is the nature of this complexity, particularly in light of the way Smartphones are now highly multi-functional. To this end the current study aims to address this gap in the literature by qualitatively exploring the lived experiences of Smartphone users, with a focus on the different ways in which they use their phones, the value that they place on these devices and their perceptions about whether they feel attached to them. We advance three research questions (RQs) to address these enquiries:

RQ1: What types of experiences, attitudes and feelings characterise users' relationships with their Smartphones?

RQ2: Do users value and make use of any specific features that their Smartphone offers?

RQ3: Do Smartphone owners feel a sense of attachment to their Smartphones and why?

\section{Method}

In order to address these research questions, an exploratory qualitative approach was used underpinned by a post-positivist epistemology. Specifically, we conducted three focus groups with 18 Smartphone owners to gain insights into the attitudes, thoughts and feelings that users hold about their devices, how and when they use them, and their views on why they may feel attached to them. Smartphones are social tools and therefore we deemed it important to utilise an interactive focus group method which would allow us to draw out perceptions of social norms in addition to the individual lived experiences of Smartphone owners.

\subsection{Participants}

A purposive opportunity sample of 18 participants who owned Smartphones were recruited via the Institute of Psychology's participant pool at the University of Wolverhampton. Therefore all participants were Undergraduate students studying Psychology. Three focus groups were held with 18 participants (14 female and 4 male) having an average age of 25.9 years. The average age with which participants owned their first mobile phone was 14.5 years. The first group consisted of 4 females; the second group had 5 females and 3 males and the third group included 5 females and 1 male. All of the participants owned a Smartphone at the time of data collection.

\subsection{Materials and Process}

A private interview room on the University campus which could comfortably accommodate up to 10 participants was used. The room was arranged so that participants were sitting in a circle 
facing one another. Prior to commencement of the focus group discussions, all participants were given an information sheet outlining the nature of the study and provided their consent to participate. All participants agreed to keep what was discussed during the focus group confidential and to protect other participants' anonymity. Prior to the focus discussions taking place the researcher established a group culture and expectations of conduct, which included no personal attacks on other members, recognising that all opinions were valid, respecting the comments made by other members even if they did not agree with their own, and talking one at a time in a clear and concise manner. In addition to asking some questions about the backgrounds of participants (including their age, sex, age at which they owned their first phone and their current phone make and brand) the focus group topic guide included a number of open-ended questions about their attitudes, thoughts and feelings about and experiences of using their phones, how and when they use them, their favourite features, and perceptions relating to their attachment to their devices. The topic guide included questions such as: "tell us a little about your Smartphone and what it means to you?", "what sort of things do you use your Smartphone for?" and "do you feel attached to your Smartphone?" The full topic guide is available upon request from the authors.

The focus groups were all held within the second week of February 2016. Each focus group concluded once the participants had discussed each question on the topic guide and were satisfied that they had said everything that they wanted to say. The first group (4 participants) lasted 39 minutes, the second group ( 8 participants) took 1 hour and 10 minutes and the third group (6 participants) concluded after 1 hour. After the three focus groups were completed, the researchers agreed that saturation had been achieved and therefore no further sessions were required. The focus group discussions were recorded on a Dictaphone and transcribed verbatim.

\subsection{Data analysis}

Following transcription the focus groups were analysed by a single experienced member of the research team using thematic analysis. The analysis followed the process recommended by Braun and Clarke (2006) and included: familiarisation with the data, generation of initial codes, searching for themes, reviewing themes, and defining and naming themes. The data were coded at the latent level, to provide an overview of the entire data set and subsequently involved refining the coded data within each theme. This ensured that each theme was mutually exclusive and the coded data and extracts accurately represented the themes. In order to 
strengthen the rigor and reliability of the study, the themes developed by the primary coder were checked by two other members of the research team and refined following discussion.

\section{Results/Discussion}

A number of themes and sub-themes were identified from participant accounts. The first theme was; "representation of phone" with two sub-themes of "anthropomorphic representation" and "materialistic representation". The next main theme was "uses", with sub-themes of "functional", "informational", "interactional/connection" and "recreational". The final two main themes related to factors which determined usage; the first of these being "uses and gratification determining usage" and secondly, "context determines usage". Each of these is discussed in the subsequent sections.

\subsection{Representation of mobile phone}

Two somewhat conflicting sub-themes which emerged from the analysis suggested the notion of Smartphones as being simultaneously "anthropomorphic" whilst also "materialistic".

\subsubsection{Anthropomorphic representation}

Many of the participants framed their discussions of their smartphones in an anthropomorphic context, in other words seemingly attaching human qualities and characteristics to these devices. This suggested that the informational functions they serve are almost "friend-like", as illustrated by the following quotes from Focus Group 1 and Focus Group 2:

“..And like Siri's my best friend, like for spelling, finding anything, like she's my best friend" (FG1P2)

"I think our phones are becoming like virtual friends really, because think back in the day, maybe when we didn't have such good, our phones didn't do so much, if you needed an answer for something you'd call someone. Maybe call someone that may know or you'd go to a book or something, but now your phone's like a virtual friend, Google, and Siri and Corona and they're like your friend....like you rely on them like you would, maybe like you would before you would have relied on calling your Mum. How'd you bake a cake? You call your Mum" (FG2P1)

In this way, participants also made reference to how this was fundamental for "survival", suggesting it to be a crucial tool in contemporary society. Specifically, Focus Group 2 illustrated two participants' agreement on this:

\section{Focus Group 2}


FG2P2: 'It's become this all important tool to get everybody through their day hasn't it, (agreement), gotta have a mobile phone with you...can't survive without it (agreement)"

FG2P1: "Like a personal therapist."

In a slightly distinct way, but still alluding to the notion of anthropomorphism, participants discussed the way in which notifications delivered through Smartphones shifted the source of dependency between themselves and the phone, in a way in which it was dependent on them (rather than vice versa as in the previous discussion):

"It's like it's crying for you" (FG2P4)

All in all, this notion of anthropomorphism appeared to be a strong drive behind the apparent attachment which participants held towards their Smartphones, specifically in developing strong emotional connections to them. In particular, Focus Group 2 included two key discourses which corresponded to this notion:

\section{Focus Group 2}

FG2P1: "Yeh I'm actually really attached and I've only just realised..."

FG2P8: "It's like having a pet"

FG2P2: "It is like a relationship or having a pet, you've got to kind of commit, it's just to what extent you're prepared to commit or engage with it."

FG2P1: "I think you're right, it is like a pet or something. Like a virtual relationship because it gives you the emotions that other people may give you, like make you angry, happy, excited, sad, uh depending on what you're doing with it. I think that maybe that's why we're so attached to them."

FG2P7: "A strange electronic parasite, (laughter, agreement)"

\section{Focus Group 2}

FG2P2: "I think whatever your relationship with them, love them or hate them, they're yours aren't they? It's part of you, so if you can't find it, it is like there's a part of you missing."

FG2P1: "Yeh, yeh you love to hate them really don't you? You love them because they make your life so much easier, and in another sense you wish you weren't so dependent in another sense."

"It's like my baby, I'd rather have a phone than a baby to be honest, they're a lot easier to take care of" (FG3P4)

\subsubsection{Materialistic representation}

In contrast to the previous sub-theme of anthropomorphic representation, participant accounts in Focus Group 1 centred on how the Smartphone itself was largely dispensable or 
disposable due to its materialistic nature. In this way, participants are alluding to the mobile device itself rather than the more abstract representations it can afford (as mentioned previously);

"I had mine insured when I bought it so, if something happens they'd just replace it." (FG1P4)

"But it's materialistic, so it'd just get replaced." (FG1P4)

In general, this appears to represent highly disparate conceptions which are afforded to Smartphones in a way which suggests users' attachments are related to the abstractive representations they hold whereas they concurrently perceive the devices themselves to be materialistic in nature and thus are not as emotionally compromised by having to replace them.

\subsection{Uses}

Four sub-themes emerged pertaining to different types of uses of Smartphones, interestingly showing distinct patterns of attachment experiences and behaviours. These uses were "functional", "informational", "interactional/connection" and "recreational", each discussed subsequently.

\subsubsection{Functional}

When reflecting on the features of their Smartphones, participants frequently mentioned features which were largely functional, such as SatNav and Maps, calculator, clock, notes, and diary functions which assist in everyday tasks:

"I think as well because I'm older I'm just in awe of the fact that I've got a camera, computer, you know, a map, everything in my pocket (laughter, unintelligible). I don't have to take out a big A to Z map anymore; it's like wow I've got one in my pocket" (FG1P3)

"SatNav, SatNav has been my new thing; I haven't bought a SatNav in years, since they've been on the mobile phone. I think, I'd imagine that is probably um equal to how I use it to surf the net, because if I've got anywhere new to go, that's how I get there" (FG1P3)

"I use it for the calculator quite a lot as well (agreement). Um, I always use the calculator and I always use it as an alarm." (FG2P1)

"I have to take it with me a lot of the time because I use it as my bus ticket as well....It's significantly cheaper for a month of travel." (FG2P7) 
Interestingly, for one participant in Focus Group 1, she identified that her attachment to her Smartphone was largely attributed to its functionality:

"I'm attached because it's my go to handy tool, but if I lost the use of that tool, it wouldn't be the end of the world because there are alternatives, that I could use. It would just take me longer than using a mobile phone, (pause) and the good thing is that we don't have to dial the house phone anymore so it doesn't take forever to do seven digits (laughter), so it wouldn't be that much of a, you know, switch" (FG1P3)

Additionally, another participant reflected that a number of functions assisted in her ability to stay organised:

"I uh, personal to me, I'm not a very organised person, so my phone I feel helps me be more organised, because there's notes on there and things like that, um and especially like the calendar. Um, I've got a diary but sometimes I forget to take that with me because I'm a bit disorganised, but I put everything in my phone, so you can check you, the diary in a sense but it also reminds you of things that are coming up (agreement) and then with your notes, if I think I'm having a really good idea or something, put it in my phone and I don't forget about it or um and I can come back to it later basically. So I think it makes me more organised as a person." (FG2P1)

\subsubsection{Informational}

In addition to functional features afforded through Smartphones, participant accounts also focused on their usage of informational resources, largely through specific websites, for health information and cooking inspiration:

"Fast processing of whatever information I want. Sometimes it might just be I'm thinking um what should I do for tea? I've got these ingredients in the fridge, ooh I'm on the phone, Google it and actually oh that looks good to cook. It's just like really quick easy." (FG1P3)

"I think the weirdest thing that I'd use mine for is self-diagnosing myself (laughter, unintelligible). Type in on Google my symptoms and I think that's, probably think I'm dead, (laughter, unintelligible) but that's probably one of the weirdest things I use it for, but I do use it a lot for that, like really hypochondriac." (FG1P2)

In both these cases, participants identify the benefits of search engines (i.e. Google) for enabling this informational function, highlighting the importance of Internet connectivity as a key resource in Smartphone usage. However, at the same time there is also some acknowledgement of the more dangerous side of constant Internet connectivity, specifically in terms of how it may fuel excessive health-checking behaviours. This adds to growing concerns 
amongst healthcare professionals about how constant checking of health symptoms online (or 'Cyberchondria' as it is sometimes known) may lead to a state of medical anxiety (e.g. see White \& Horvitz, 2009).

\title{
3.2.3. Interactional/Connection
}

A further key feature of smartphones was their ability to afford connection with others, both "abstractly" by having contact details of others available (e.g., contacts/phone book) but also through more concrete interactional connections through messaging services and apps (e.g. WhatsApp groups). Focus Group 2 for example, included insight into how participants experienced "panic" or emotional disharmony with the thought of not having contact information for others which they would typically have in their Smartphone:

\begin{abstract}
Focus Group 2
FG2P2: "I'm bad if I, if I forget it. I panic then, because then I've lost contact, I've got no way of contacting anybody, (...) I'm bad if I've left it at home. I will go back home and fetch it, so I am sometimes late for stuff because I've had to go back for my phone." FG2P1: "A connection yeh,(...) yeh yeh, it's just having it there."

FG2P7: "...knowing that they can or that you can..."

FG2P1: "yeh, you feel like everyone's with you if you have your phone"
\end{abstract}

\section{Focus Group 3}

"I think mostly because I used to live with my grandparents and then when I came to uni, my grandad was left on his own obviously, so my phone is mainly to make sure that my grandad is ok. So if he has anything like, my aunty or my mum or my grandad will contact me, and then that's usually what it's there for. Obviously I use it for like everything else, but that is like the main focus of having a phone. I can't not have a phone for that reason." (FG3P4)

In a slightly distinct way, other participants drew on the established connections and interactions available through systems on their Smartphones, which they would otherwise be without and thus feel "disconnected":

"Yeh, like I have like five different Whatsapp groups and five different conversations going on and I keep in touch with my sister who lives in Peterborough, and you know you, you've got groups for uni stuff, you got groups of friends. I, I'd just feel really disconnected, because it is my, my way of keeping in touch with my friends and family who I don't live with.” (FG1P1).

\section{Focus Group 3}

FG3P5: "I think it's mostly just staying in contact with people, because I always message people like all the time, and if I didn't have a phone I couldn't do that."

FG3P4: "It's a lot easier to like make plans and stuff, (...) do you want to meet up or do you want to go and do this like ok? And you can tell them like where you are, where 
you're gonna meet, when you're gonna meet, and it's a lot easier, otherwise you'd have to like go to them and find out through someone else or it's just a lot easier."

\subsubsection{Recreational}

A distinct sub-theme of the "uses" theme, was the notion of Smartphones affording "recreational" uses. That is, this represents how phones offer leisure or entertainment opportunities, such as playing digital games, or listening to music:

\section{Focus Group 1}

FG1P3: "Candy Crush used to be my thing but I got over it."

R: "No longer addicted to Candy Crush?"

FG1P3: "I don't have time, not with um assignments to do."

FG1P1: "Uh I'm addicted to, I've got a new inside out game, like a bubble game, you pop the bubbles out, um..."

FG1P2: "I've got a few routine games that I play."

Interestingly for a participant in Focus Group 2, he explicitly states that he does not use his Smartphone for what may be deemed to be its primary purpose (i.e. making phone calls) and only uses it for its recreational features:

"I don't even send calls or texts, I just listen to music on it (laughter), that's it." (FG2P6)

Taking this notion, as well as these sub-themes collectively, this does raise the question about whether the term Smart "phone" is still a current representation of its full potential and uses. Indeed, among this theme, there was relatively little discussion about phone calls, highlighting that its designed purpose for these users is not the most readily used function. It would be useful to consider whether "mobile device" is a more representative term which should be used, given that its usage is largely similar to other "mobile devices" such as tablets (e.g., iPads) rather than traditional telephones. Further, the fact that Smartphone users make use of these devices in such diverse and personal ways reflects similar discussions around the unique ways in which people consume the Internet (e.g. see Orchard \& Fullwood, 2010). Further research may wish to explore individual difference factors (e.g. personality, sex and age) and how these may influence different patterns of Smartphone feature use.

\subsection{Uses/Gratifications determine usage}

When reflecting on Smartphone use, participants appeared to have a number of different uses and gratifications to fulfil, which was the main determining factor for what features were used 
and how much it was used. That is, two sub-themes on this were using for "alleviating boredom" and purely out of "habituation", discussed below.

\subsubsection{Alleviating boredom}

In respect of alleviating boredom, participants discussed how this was particularly relevant for their use of recreational (e.g., listening to music) and connection features (being "in the loop" on Facebook):

\section{Focus Group 1}

FG1P4: "I use it when I'm on bored, (agreement) it's usually on the train, or the bus." FG1P2: "I have like a rit, not a ritual, like on the bus on the way here, always listen to music, or, like I've always got it in my hand (unintelligible) just sat there doing nothing. So I just, the only time I can say I definitely have my phone in use, um, but other than that it's just, when and wherever really."

"Yeh, um, yeh, I just use it whenever, mainly boredom I think sometimes." (FG1P1)

"I just like generally being nosey, because you get bored and you can just sit on like Facebook and see what everybody else is doing. You don't have to like contact them but you can just, have a scroll through and see what everyone's doing" (FG3P4)

This suggests these uses, in comparison to "informational" or "functional" ones which are used in more targeted-driven ways for more strategic uses, are largely a "time-wasting" activity to alleviate boredom. In order to understand technological behaviours, such as Smartphone use, it is therefore essential to underpin this with a nuanced account of what activities and affordances are driving behaviour rather than simply looking at behaviour per se (e.g., time spent on mobiles) or the outcome of that behaviour (e.g. how it makes someone feel). This has key implications for so-called Smartphone addiction and associated measurements of this, which do not provide such as account of these underpinnings and therefore may not be able to draw useful practical intervention strategies. Indeed, current Smartphone addiction scales tend to focus on the personal and social outcomes of using these devices excessively (e.g. how this might affect relationships or how someone functions at work), ones preoccupation with the device and how much it dominates their thought processes, negative emotional consequences of not having access to the device (e.g. withdrawal), the proportion of one's time spent using the device and efforts that may have been made to curb excessive usage (e.g. tolerance) (e.g. see Kwon et al, 2013; Kim et al., 2014). In this sense, one might make a similar argument to that which has been forwarded by some scholars regarding Internet addiction. Specially, maladaptive behaviours would still occur in the absence of the technology (e.g. problematic gamblers would gamble offline instead) and that it may not necessarily be the technology as a 
whole that users are addicted to, but rather very specific functions or features that are accessed via them (e.g. see Stern, 1999; Widyanto \& Griffiths, 2006).

\subsubsection{Habituation}

Another sub-theme on Uses and Gratifications emerged from participants' discussions about how their Smartphone use had become habituated. That is, Focus Group 3, in particular, reflected upon how their psychological attachment to their phones was largely to do with the way in which using it had become a "habit". This was discussed in reference to having a phone "to hand" and also the largely automatic behaviours of swiping to check for the time;

\section{Focus Group 3}

FG2P2: "I do use my phone a little bit when it's on charge, (...) my phone, probably shouldn't because it's like plugged into the wall and I've also got an e-cigarette and when that's plugged into the wall I probably shouldn't use that either, because I dunno it feels a bit wrong. It's plugged into the wall, you don't really wanna be like taking a drag on it, so, I dunno but I think I'm attached to my e-cigarette as I am to my phone because I've constantly got them both in my hand, so I'm like walking on, toking it like (inhale) you know (...) constantly"

FG3P6: "(...) smoking a phone"

FG3P2: "But I think um they're kind of on the same par like. If I misplace my phone or my e-cigarette I start getting like really hot, like oh my god where is it, where is it, where is it? And then like you have a panic attack and I dunno (...) lose my cigarette (...) I think it's kind of worse when I lose my e-cigarette, then my phone which is quite bad...."

\section{Focus Group 3}

FG3P5: "I think we check out of habit as well though because I check my phone for the time like so many times and not actually looked at the time"

FG3P4: "(...) unlocked it then put it down and like I haven't even checked the time" FG3P5: "Yeh it's just habit"

\subsection{Context determines usage}

In contrast to the previous theme relating to "habituation", participants also reflected upon their Smartphone usage as being largely determined by their immediate context or situation. Specifically, Focus Group 2 discussed how the context determined what features were used and when:

\section{Focus Group 2}

R: "Would you find you're more likely to use your phone on like specific occasions? On, on certain occasions rather than others?"

(Resounding yes). 
FG2P1: "If I was in the house I probably wouldn't sit with the phone, I always lose it, but then if I was out of the house I'd probably be on there, taking pictures, (...)"

FG2P3: "Find out where people are and stuff"

FG2P1: "I think when you're out the house you want it close to you just in case you need it in emergencies as well."

FG2P7: "If you're on a day out you might use the camera on it as well"

FG2P4: "You can use it anywhere you go, just in certain situations you use it more, but you can use it anywhere"

FG2P3: "It's like saying that we lose our confidence when we leave out the house, so we have to constantly look, rely on our phone like, I dunno..."

Here it is apparent that the "connection" usage is particularly relevant for when users are not in their home environment or a secure-base and thus feel a need to have connection with others. This largely reflects the notions asserted in attachment theory (Ainsworth \& Bell, 1970), in which individuals who are insecurely attached may use their attachment agent as a securebase in which to explore their environment but also experience distress or anxiety upon separation. Thus, the way in which individuals' Smartphones function as a connection agent enables them to feel secure when away from the familiar, home setting. The tendency to check devices while alone or in an unstimulating environment also seems to be reminiscent of findings from a recent study by Wilson et al. (2014), which found that participants preferred engaging in mundane activities or exposing themselves to electric shocks to being left alone for 6 to 15 minutes with just their own thoughts. Although somewhat speculative, living in an age where we are perpetually connected to the thoughts of others (e.g. via social media) or always have technology at our disposal to relieve boredom, may be creating the conditions in which we have become used to not being left alone with our thoughts.

Additionally, participants were aware of the social etiquette associated with Smartphone use and reflected upon particular situations in which they would avoid using their phones:

\section{Focus Group 2}

FG2P8: "I think that, I think when there's certain setting where I wouldn't look at my phone as much just because I'm just more, the phone is less entertaining, than what's going on so like my Nan's house, my Nan's house when I go there is always quite busy, like the whole family's there and it's just fun so, I don't really look at my phone because I'm doing something else or I'm actually like talking to people (yeh)."

FG2P2: “(...) engaging with real people not on the phone."

"I think it depends how like important it is, if you only like, right now all our phones are in our bags because this is kind of important but if it wasn't (someone else talking...silent...) yeh, we know we have to" (FG3P5) 
"I think in certain situations you just can't like use a phone, (...) you know when you're like in an interview, even if you were getting a call then you probably couldn't answer it, because you just think, I'm not gonna get this job if I pick up the phone half way through. I think it just depends on the situation really" (FG3P2)

This seems rather distinct from the earlier theme relating to habituation in which, particularly in some cases, Smartphone use appeared to be automatically-driven to serve a need gratification. Conversely, here it is more apparent that participants are mindful of their usage, in respect of the social context in which they are residing.

\section{Overall Discussion and Conclusions}

A number of interesting findings were evident from the accounts of focus group participants. Unsurprisingly, Smartphones were reported to be so much more than devices to make calls or send texts on. To our participants they also functioned as alarm clocks, media players, calculators, personal assistants, satellite navigation systems, Internet browsers, cameras and devices to play games on. One participant even went as far as to state that his phone was never used for the primary purpose it was designed for (i.e. communicating with others) but rather was almost exclusively used for listening to music. Evidence from this study would also suggest that users do not user their phones in a uniform manner but rather have very personal and unique patterns of Smartphone consumption. This 'individualised' perspective on Smartphone usage fits well within the framework of the Uses and Gratifications model (Katz et al., 1974), which argues that users make media choice decisions in order to gratify very personal needs. What is unclear is which particular 'uses' are most likely to be associated with which specific 'gratifications'. Although primary motivations for using Smartphones include escaping problems, staying connected and relieving boredom (Chen \& Katz, 2009; Pew Research Center, 2015; Smetaniuk, 2014; Walsh et al 2008, 2009), future research may wish to explore potential links between individual motivations and the specific Smartphone functions used to gratify them. For example, might someone who is motivated to relieve boredom be more likely to use the entertainment features of their phone?

The multifunctional nature of Smartphones has a number of implications for the assessment of attachment or addiction to these devices. Specifically, it brings into sharp focus the debate around whether users are addicted or attached to the device itself, or to the different purpose(s) for which they are using it to serve. Indeed, numerous scholars have put forward similar arguments with regards to the addictions to the Internet versus addictions on the Internet debate. 
In other words, it has been argued that users may be addicted to very specific online activities (e.g. social media, online gaming) rather than the Internet as a whole. Furthermore, in the absence of the Internet, users would find other avenues to fuel their addictions (Stern, 1999; Widyanto \& Griffiths, 2006). For example, the 'addicted' online gambler would visit an offline bookmaker instead. It may be that Smartphone addiction operates in much the same way and we would argue that assessment tools need to pay more heed to the manner in which individuals make use of these devices in order to gather a more nuanced account of the precise nature of addictive tendencies. It may be that certain patterns of Smartphone usage are more likely to lead to dependency on or attachment to the device.

Perceived attachment to Smartphones was reflected in many of the participants' accounts, both in terms of an emotional attachment (e.g. "...our phones are becoming like virtual friends"; “...a virtual relationship because it gives you the emotions that other people may give you, like make you angry, happy, excited, sad”), in some users' habitual use of these devices (e.g. "...I check my phone for the time like so many times and not actually looked at the time") and the feelings of anxiety expressed at the thought of losing their phones (e.g. "If I misplace my phone...I start getting like really hot, like oh my god where is it, where is it, where is it? And then like you have a panic attack"). Rubin (1984) has noted that users express greater affinity with a medium when it is used in a ritualised manner, because habitual use would normally reflect diversionary activities such as companionship and relaxation. However, more goalorientated use, for example gratifying informational needs, would not create such an affinity. The fact that many participants described using their Smartphones to relieve boredom, to entertain themselves, to stay connected or to help them feel relaxed when they were outside of their comfort zone may go some way towards explaining why an emotional affinity to these devices develop as well as the sense of discomfort which is experienced when they are not in their possession. Future research may wish to explore the social and developmental implications of persistent phone-checking when in unfamiliar or uncomfortable surroundings. For example, does using the phone as a 'crutch' when one is alone or feeling insecure hinder one's interactions with others and the environment or prevent people from building resilience through facing challenging situations?

Participants also described their Smartphones as being disposable and materialistic but at the same time they made anthropomorphic and sentimental associations with them. Although it might appear problematic to reconcile these opposing viewpoints, what this might suggest is 
that users are not necessarily attached to the device itself, but rather the affordances that it provides them. Indeed, photos, contacts and other information downloaded on to the device can be transferred over to a replacement phone with relative ease. Thus, the features which allow users to personalise the phone and reflect an aspect of the self (e.g. being able to store personal memories; Meschtscherjakov, 2009; Tian et al., 2009, Venta et al., 2008) are transferable and not locked in to one specific device. Individuals may be less inclined to perceive Smartphones as status symbols, given that even the most desirable phones can be acquired on monthly contract plans. The latest and most wanted Smartphones are not necessarily out of reach to the average person; they are no longer exclusive gadgets for the privileged minority.

Overall, the findings from this study provide a rich account of the relationship that some users have with their Smartphones and should provide a stepping stone for future research to explore in more detail the manner in which the varying affordances offered by this technology might shape our perceptions of and attachments to them. The study was not without its limitations however, as it focused on the accounts of UK Smartphone owners who were all Undergraduate students. Although the conclusions from this research cannot necessarily be applied to other situations and populations, further research may wish to explore these phenomena using similar methods but in different environments (e.g. different cultures and age groups) as well as complementary methods (e.g. interviews) to gather individual accounts and thus eliminating the potential for groupthink. Triangulating data sources through the accumulation of such findings may enable a more comprehensive picture of the relationship that users have with their smartphones to emerge (Shenton, 2004).

\section{References}

Ainsworth, M. D. S., \& Bell, S. M. (1970). Attachment, exploration, and separation: Illustrated by the behavior of one-year-olds in a strange situation. Child Development, 41, 49-67.

Aoki, K. \& Downes, E.J. (2003). An analysis of young people's use of and attitudes toward cell phones. Telematics and Informatics, 20 (4). Elsevier: 349-64.

BBC News (2012). UK's first 4G mobile service launched in 11 cities by EE. Retrieved $9^{\text {th }}$ January 2017 from http://www.bbc.co.uk/news/technology-20121025

Chen, Y \& Katz, J.E. (2009). Extending family to school life: College students' use of the mobile phone. International Journal of Human-Computer Studies, 67, 179-191

Choi, H.S., Lee H.K., \& Ha, J. (2012). The influence of smartphone addiction on mental health, campus life and personal relations - Focusing on K university students. Journal of Korean Data \& Information Science Society, 23(5), 1005-1015. 
Fullwood, C. (2015). The role of personality in online self-presentation. In A. Attrill (Ed.) Cyberpsychology (pp. 9-28). Oxford University Press, Oxford.

Haug, S., Castro, R. P., Kwon, M., Filler, A., Kowatsch, T., \& Schaub, M. P. (2015). Smartphone use and smartphone addiction among young people in Switzerland. Journal of Behavioral Addictions, 4, 299-307.

Head, M. \& Ziolkowski, N. (2012). Understanding student attitudes of mobile phone features: Rethinking adoption through conjoint, cluster and SEM analyses. Computers in Human Behavior, 28, 2331-2339

Jarvenpaa, S.L. \& Lang, K.R. (2005). Managing the paradoxes of mobile technology. Information System Management, 22(4), 7-23

Joo, J., \& Sang, Y., (2013). Exploring Koreans' smartphone usage: an integrated model of the technology acceptance model and uses and gratifications theory. Computers in Human Behavior, 29, 2512-2518.

Katz, E., Blumler, J.G., \& Gurevitch, M. (1974). Utilization of mass communication by the individual. In: Blumler JG and Katz E (eds) The Uses of Mass Communications: Current Perspectives on Gratifications Research. Beverly Hills, CA: SAGE, pp. 19-32.

Kim D, Lee Y, Lee J, Nam JK, Chung Y. (2014). Development of Korean smartphone addiction proneness scale for youth. PLOS ONE, 9(5).

Kwon, M., Lee, J., Won, W., Park, J., Min, J., Hahn, C. et al (2013) Development and validation of a smartphone addiction scale. PLoS One, 8(2), 1-7.

Leung, L., \& Wei, R. (2000). More than just talk on the move: Uses and gratifications of the cellular phone. Journalism and Mass Communication Quarterly, 77(2), 308-320.

Livaditi, J., Vassilopoulou, K., Lougos, C., \& Chorianopoulos, K. (2003). Needs and gratifications for interactive TV applications: Implications for designers. In Proceedings of the 36th Hawaii international conference on system sciences.

Lopez-Fernandez, O. (2015). Short version of the Smartphone Addiction Scale adapted to Spanish and French: Towards a cross-cultural research in problematic mobile phone use. Addictive Behaviors, 64, 275-280.

Madell, D.E. \& Muncer, S. (2007). Control over social interactions: An important reason for young people's use of the internet and mobile phones for communication? Cyberpsychology \& Behavior: The Impact of the Internet, Multimedia and Virtual Reality on Behavior and Society, 10(1), 137-40.

Meschtscherjakov, A. (2009). Mobile attachment: emotional attachment towards mobile devices and services. In Proceedings of the 11th International Conference on HumanComputer Interaction with Mobile Devices and Services, 102:1-102:1. MobileHCI '09. New York, NY, USA: ACM.

Orchard, L.J. \& Fullwood, C. (2010). Current perspectives on personality and Internet use. Social Science Computer Review, 28(2), 155-169.

OFCOM (n.d.) Fast Facts. Retrieved $9^{\text {th }}$ January from https://www.ofcom.org.uk/aboutofcom/latest/media/facts

OFCOM (2016) Adults' Media Use and Attitudes Report 2016. Retrieved $9^{\text {th }}$ January from https://www.ofcom.org.uk/_data/assets/pdf_file/0023/82265/section-4-digital-mediatake-up-and-use.pdf 
Pew Research Center (2015). Technology Device Ownership, 2015. Retrieved $9^{\text {th }}$ January from file://C:/Users/Sally/Downloads/PI_2015-10-29_device-ownership_FINAL.pdf

Rubin, A. M. (1984). Ritualized and instrumental television viewing. Journal of Communication, 34(3), 67-77.

Samaha, M., \& Hawi, N. S. (2016). Relationships among smartphone addiction, stress, academic performance, and satisfaction with life. Computers in Human Behavior, 57, 321-325. Shenton, A.K. (2004). Strategies for ensuring trustworthiness in qualitative research projects. Education for Information, 22, 63-75.

Stern, S. (1999). Addiction to technologies: A social psychological perspective on Internet addiction. Cyberpsychology, Behavior and Social Networking, 2(5), 419-424.

Smetaniuk, P. (2014). A preliminary investigation into the prevalence and prediction of problematic cell phone use. Journal of Behavioral Addictions 3(1): 41-53.

Srivastava, L. (2005). Mobilephones and the evolution of social behaviour. Behaviour \& Information Technology 24(2), 111-29.

Tajfel, H., \& Turner, J. C. (1979). An integrative theory of intergroup conflict. In W. G. Austin \& S. Worchel (Eds.), The social psychology of intergroup relations: 33-47. Monterey, CA: Brooks-Cole.

Tian, L., Junqi S., and Yang, Z. (2009). Why does half the world's population have a mobile phone? An examination of consumers' attitudes toward mobile phones. Cyberpsychology \& Behavior: The Impact of the Internet, Multimedia and Virtual Reality on Behavior and Society 12(5), 513-16.

Toda, M., Monden, K., Kubo, K., \& Morimoto, K. (2006). Mobile phone dependence and health-related lifestyle of University students. Social Behavior and Personality: An International Journal, 34(10), 1277-84.

Vanden Abeele, M., .Antheunis, M.L. \& Schouten, A.P. (2013). Me, myself and my mobile: A segmentation of youths based on their attitudes towards the mobile phone as a status instrument. Telematics and Informatics, 31(2): 194-208.

Van Deursen, A. J. A. M., Bolle, C. L., Hegner, S., \& Kommers, P. A. M. (2015). Modeling habitual and addictive smartphone behavior: The role of smartphone usage types, emotional intelligence, social stress, self-regulation, age, and gender. Computers in Human Behavior, 45, 411-420

Venta, L., Isomursu, M., Ahtinen, A \& Ramiah, S. (2008). 'My phone is a part of my soul'-How people bond with their mobile phones. In Mobile Ubiquitous Computing, Systems, Services and Technologies, 2008. UBICOMM'08. The Second International Conference on, 311-17. IEEE.

Walsh, S. P., White, K.M. \& Young, R.M. (2008). Over-Connected? A qualitative exploration of the relationship between Australian youth and their mobile phones. Journal of Adolescence, 31(1), 77-92.

Walsh, S. P., White, K.M. \& Young, R.M. (2009). The phone connection: A qualitative exploration of how belongingness and social identification relate to mobile phone use amongst Australian youth. Journal of Community \& Applied Social Psychology, 19(3), 225-40.

Wang, D., Xiang, Z., \& Fesenmaier, D.R. (2014). Adapting to the mobile world: A model of smartphone use. Annals of Tourism Research, 48, 11-26.

White, R.W. and Horvitz, E. (2009). Cyberchondria: studies of the escalation of medical concerns in web search. ACM TOIS, 27(4), 23. 
Widyanto, L., \& Griffiths, M. (2006). Internet addiction: A critical review. International Journal of Mental Health and Addiction, 4(1), 31-51.

Wilson, T. D., Reinhard, D. A., Westgate, E. C., Gilbert, D. T., Ellerbeck, N., Hahn, C., Brown, C.L., \& Shaked, A. (2014). Just think: The challenges of the disengaged mind. Science, 345, $75-77$. 